\title{
Changes in pH affects Bioactivity of Chitosans from Callinectes sapidus
}

\author{
*OMOGBAI, BA; IKENEBOMEH, MJ; OBAZENU, EI; IMONI, AA
}

Department of Microbiology, Food and Industrial Division, Faculty of Life Sciences, University of Benin, Benin City, Nigeria

*Corresponding Authors Email: barry.omogbai@uniben.edu, Tel: +2348023579328

\begin{abstract}
The effect of $\mathrm{pH}$ on the antimicrobial activity of chitosans was evaluated with five different molecular weight chitosans (DMPAC: $\mathrm{M}_{\mathrm{W}}$ 152; DMPCA: $\mathrm{M}_{\mathrm{W}}$ 338; DPMCA $_{(2)}: \mathrm{M}_{\mathrm{W}} 418 ;$ DMCPA: $\mathrm{M}_{\mathrm{W}} 550$ and DCMPA, $\left.\mathrm{M}_{\mathrm{W}} 558\right)$ at $500 \mu \mathrm{g} / \mathrm{ml}$ concentration on different food-borne bacteria and fungi. Studies on $\mathrm{pH}$ was carried out at $\mathrm{pH} 3.0,3.5,4.0,5.0$ and 5.8 with $500 \mu \mathrm{g} / \mathrm{ml}$ chitosan using an overnight broth of bacteria ( $0.05 \mathrm{ml})$ sub-cultured in nutrient broth or MRS broth (for lactic acid bacteria). Fungi were incubated at $28 \pm 2^{\circ} \mathrm{C}$ for $72 \mathrm{~h}$ and enumerated on sabouraud dextrose agar. The viable cell count of Staphylococcus aureus at $\mathrm{pH} 3.0$ for all chitosans ranged between $1.23-1.76 \log _{10} \mathrm{CFU} / \mathrm{ml}$ while at $\mathrm{pH} 5.8$ viable cell numbers was $2.38-5.26 \log _{10} \mathrm{CFU} / \mathrm{ml}$ compared to the initial inoculum number of 7.06 $\log _{10} \mathrm{CFU} / \mathrm{ml}$. The growth of Listeria monocytogenes was totally suppressed by $500 \mathrm{ug} / \mathrm{ml} \mathrm{chitosan}$ at or below $\mathrm{pH}$ 5.0. Bacillus subtilis was susceptible to inhibition at low $\mathrm{pH}$ and had no detectable viable cell counts at $\mathrm{pH}$ 3.0-4.0. The viable cell numbers of Escherichia coli 0157:pH7 were reduced by approximately $2 \log _{10}$ cycles at $\mathrm{pH} 5.8$ and by more than $5 \operatorname{logs}$ at pH3.0 with DMPAC chitosan. Rhizopus Stolonifer was reduced to non-detectable levels by DMPAC chitosan at $\mathrm{pH} 3-3.5$.This mould was more sensitive to chitosan $(500 \mu \mathrm{g} / \mathrm{ml})$ at all $\mathrm{pH}$ compared to Penicillium expansum and Aspergillu sniger. Saccharomyces cerevisiae and Pichia fermentans were similarly affected by low $\mathrm{pH}$. The results of the present study show that application of chitosan to acidic foods such as fruit juices will enhance its effectiveness as a natural preservative.
\end{abstract}

\section{DOI: https://dx.doi.org/10.4314/jasem.v23i3.7}

Copyright: Copyright (C) 2019 Omogbai et al. This is an open access article distributed under the Creative Commons Attribution License (CCL), which permits unrestricted use, distribution, and reproduction in any medium, provided the original work is properly cited.

Dates: Received: 07 February 2019; Revised: 21 March 2019; Accepted 27 March 2019

Keywords: Antimicrobial, chitosans, Crab, Acidity, Microorganisms

Chitosan is a natural polysaccharide comprising copolymer of glucosamine and $\mathrm{N}$-acetylglucosamine. It can be obtained by the deacetylation of chitin from crustacean shells. (No and Meyers, 1989). Chitin and chitosan have very similar chemical structures. Chitin exhibits structural similarity to cellulose and differs from it with the replacement of C-2 hydroxyl residues by acetamide groups (Kurita, 1998). Chitin can be transformed into chitosan that has free amino groups by removing acetyl groups $\left(\mathrm{CH}_{3}-\mathrm{CO}\right)$ from chitin molecules. Thus chitosan is the deacetylated form of chitin, meaning that the acetamide groups $\left(\mathrm{CH}_{3} \mathrm{CO}-\mathrm{NH}\right)$ in chitin are substituted into amino groups $\left(-\mathrm{NH}_{2}\right)$ in chitosan (Sakthivel et al., 2015). Hsu et al. (2002) reported that chitosan is insoluble in water, alkali and organic solvents, but soluble in most diluted acids with $\mathrm{pH}$ less than 6 . When chitosan is dissolved in an acid solution, it becomes a cationic polymer due to the protonation of free amino groups on the $\mathrm{C}-2$ position of the pyranose ring. The cationic properties of chitosan in acidic solutions give it the ability to interact readily with negatively charged molecules such as lipids and cholesterol. In this respect, chitin and chitosan have attained increasing commercial interest as suitable resource materials due to their excellent properties including biocompatibility, biodegradability, absorption, ability to form films and to chelate metal ions ( $\mathrm{Li}$ et al., 1992).Chitosan have been reported to have antimicrobial activity (Omogbai and
Ikenebomeh,2013; Sakthivel et al., 2015).In the light of the above, this paper examines the effect of changes in acidic $\mathrm{pH}$ on the antimicrobial properties of chitosan.

\section{MATERIALS AND METHODS}

Source of microorganisms: The microorganisms used in the study were bacteria including Salmonella typhimurium, Escherichia coli 0157:H7, Pseudomonas fluorescens, Vibrio parahaemolyticus and Listeria monocytogenes which were stool isolates obtained from Nigerian Institute of Medical Research (NIMR), Lagos, Nigeria. Staphylococcus aureus $\left(S A U B T_{1}\right)$, was a clinical wound isolate obtained from the Department of Medical Microbiology, University of Benin Teaching Hospital, Benin City, Nigeria. Bacillus subtilis, Lactobacillus casei and Lactobacillus plantarum were obtained from fruit wastes. Microorganisms were characterized based on shape, size and colour of colony and inspected by light microscopy. The bacteria were Gram-stained (Roberts et al., 1995). Phenotypic profiling of both Gram-positive and Gram-negative bacteria was undertaken using API 50CHB and API 20E strips (BioMerieux, Marsielle, France) respectively. Fungi (Saccharomyce scerevisiae, Pichia fermentans, Penicillium expansum, Aspergillus niger and Rhizopus stolonifer) employed in the studies were 
isolated from tropical fruits wastes of pineapple and watermelon.

Source of chitosan: This was obtained from Callinectes sapidus by unconventional methods outlined by Omogbai and Ikenebomeh (2016).

Effect of $p H$ on the antimicrobial activity of chitosan: modified method of Youssuf and Munir (2007) was employed. The effect of $\mathrm{pH}$ on the antimicrobial activity of chitosans was evaluated with five different molecular weight chitosans (DMPAC, $\mathrm{M}_{\mathrm{W}}$ 152; DMPCA, $\mathrm{M}_{\mathrm{W}}$ 338; $\mathrm{DPMCA}_{(2)}, \mathrm{M}_{\mathrm{W}}$ 418; $\mathrm{DMCPA}_{\mathrm{W}}$, $\left.550 ; \mathrm{DCMPA}, \mathrm{M}_{\mathrm{W}} \quad 558\right)$ at a $0.05 \% \quad(500 \mu \mathrm{g} / \mathrm{ml})$ concentration on different food-borne bacteria and fungi. The upper $\mathrm{pH}$ value studied was limited to 5.8 because chitosan is soluble in most organic acid solutions with less than pH 6 (Muzzarelli, 1973). Studies on $\mathrm{pH}$ were carried out at $\mathrm{pH} 3.0,3.5,4.0$, 5.0 and 5.8. The solutions were adjusted to these $\mathrm{pH}$ values with $1 \mathrm{~N} \mathrm{HCL}$ and $1 \mathrm{~N} \mathrm{NaOH}$. Then $0.05 \mathrm{ml}$ of overnight broth of each microorganism subcultured in nutrient broth or MRS broth (for lactic acid bacteria) were inoculated into $10 \mathrm{ml}$ of nutrient broth or MRS broth containing $0.05 \%$ chitosan and incubated at $37^{\circ} \mathrm{C}$ for $24 \mathrm{~h}$. Viable cells were enumerated on nutrient agar or MRS agar by pour plating $1 \mathrm{ml}$ after serial dilutions of the chitosan solutions followed by incubation at $37^{\circ} \mathrm{C}$ for $24 \mathrm{~h}$.Fungi were enumerated on sabouraud dextrose agar and incubated at $28 \pm 2^{\circ} \mathrm{C}$ for $72 \mathrm{~h}$.

\section{RESULTS AND DISCUSSION}

The effects of $\mathrm{pH}$ 3.0-5.8 on antimicrobial activity of crab chitosans are shown in Tables 1-3. The effect of $\mathrm{pH}$ on the antibacterial activity of chitosans was evaluated with five different molecular weight chitosans at $0.05 \%(500 \mu \mathrm{g} / \mathrm{ml})$ concentration on five Gram-positive bacteria (Table 1).As shown in Table 1 , the antibacterial activity of chitosan was affected by $\mathrm{pH}$, with greater activity at lower $\mathrm{pH}$ values. The viable cell count of Staphylococcus aureusat $\mathrm{pH} 3.0$ for all chitosans ranged between 1.23$1.76 \log _{10} \mathrm{CFU} / \mathrm{ml}$ while at $\mathrm{pH} 5.8$ viable cell numbers was $2.38-5.26 \log _{10} \mathrm{CFU} / \mathrm{ml}$ compared to the initial inoculum number of $7.06 \log _{10} \mathrm{CFU} / \mathrm{ml}$. Among the chitosans, DMPAC had the lowest viable cell count of $1.23 \log _{10} \mathrm{CFU} / \mathrm{ml}$ at $\mathrm{pH} 3.0$. Chitosan DCMPA had the highest viable cell count $\left(5.26 \log _{10} \mathrm{CFU} / \mathrm{ml}\right)$ for this organism at $\mathrm{pH}$ 5.8. The growth of Listeria monocytogenes was totally suppressed by $500 \mu \mathrm{g} / \mathrm{ml}$ chitosan at or below pH 5.0. Bacillus subtilis was susceptible to inhibition at low $\mathrm{pH}$ and had no detectable viable cell counts at $\mathrm{pH}$ 3.0-4.0 using $500 \mu \mathrm{g} / \mathrm{ml}$ with chitosans DMPAC, DMPCA, DPMCA (2) and DMCPA. The chitosan DCMPA although suppressed the growth of this organism had viable cell numbers of
$1.18 \log _{10} \mathrm{CFU} / \mathrm{ml}$ at $\mathrm{pH} 3.0$ and $4.06 \log _{10} \mathrm{CFU} / \mathrm{ml}$ at pH 5.8.

Lactobacillus casei and Lactobacillus plantarum also had their cell numbers reduced at low $\mathrm{pH}$ than at higher pH.At $\mathrm{pH} 3.0$ the viable cell numbers of Lactobacillus casei ranged between 1.08$2.03 \log _{10} \mathrm{CFU} / \mathrm{ml}, 1.21-2.16 \log _{10} \mathrm{CFU} / \mathrm{ml}$ (at $\mathrm{pH}$ 4.0) and 2.60-4.03 $\log _{10} \mathrm{CFU} / \mathrm{ml}$ (at $\mathrm{pH}$ 5.8). The antibacterial activity of all chitosans was stronger with decrease in $\mathrm{pH}$ against Lactobacillus plantarum. At pH 3.0 and 3.5 no viable cells were detected for DMPAC chitosan, $2.05 \log _{10} \mathrm{CFU} / \mathrm{ml}$ at $\mathrm{pH} 4.0$, and $2.52 \log _{10} \mathrm{CFU} / \mathrm{ml}$ at $\mathrm{pH}$ 5.8. Thus for all grampositive bacteria tested, the lower the $\mathrm{pH}$, the greater the antimicrobial activity of all chitosans used.

The effect of $\mathrm{pH}$ on the antibacterial activity of chitosans on Gram-negative bacteria is illustrated in Table 2. The most antimicrobial activity was found at low $\mathrm{pH}$ values. The viable cell numbers of Escherichia coli 0157:H7 were reduced by approximately $2 \log _{10}$ cycles $\mathrm{pH} 5.8$ and by more than 5 logs at pH3.0 with DMPAC chitosan. The range of viable cell reduction by chitosans at $\mathrm{pH} 3.0$ for Escherichia coli 0157:H7 was 2.052.42 $\log _{10} \mathrm{CFU} / \mathrm{ml}, 3.30-3.62 \log _{10} \mathrm{CFU} / \mathrm{ml}(\mathrm{pH} 3.5)$, 4.65-4.92 $\log _{10} \mathrm{CFU} / \mathrm{ml} \quad(\mathrm{pH} \quad 4.0), \quad 5.32-$ $5.83 \log _{10} \mathrm{CFU} / \mathrm{ml} \quad(\mathrm{pH} 5.0) \quad$ and 5.68 $5.95 \log _{10} \mathrm{CFU} / \mathrm{ml}$ ( $\mathrm{pH} 5.8$ ) respectively (Table 2 ). The viable cell numbers of Salmonella typhimuriumin a control experiment at $\mathrm{pH} 3.0$ increased from $7.57-9.23 \log _{10} \mathrm{CFU} / \mathrm{ml}$. The addition of chitosans to the medium caused a reduction in the viable cell count in the range $2.08-2.61 \log _{10} \mathrm{CFU} / \mathrm{ml}$. At $\mathrm{pH} 3.5$, the viable cell count also reduced to 3.41 4.82 $\log _{10} \mathrm{CFU} / \mathrm{ml}$. With DMPAC chitosan, Salmonella typhimurium was reduced by approximately $5.5 \mathrm{log}$ cycle at $\mathrm{pH} 3.0$ compared to 2 $\log$ cycle at $\mathrm{pH}$ 5.8.The growth of Pseudomonas fluorescens and Vibrio parahaemolyticus were similarly affected by low acidic $\mathrm{pH}$ values. With DMPCA chitosan addition at $\mathrm{pH} 3.0$ the viable cell count of these bacteria was reduced to non-detectable levels but at $\mathrm{pH} 5.8$ the viable cell counts were reduced to 1.94 and $3.22 \log _{10} \mathrm{CFU} / \mathrm{ml}$ respectively (Table 2).

The effect of $\mathrm{pH}$ on the antifungal activity of chitosan on yeast and moulds is shown in Table 3. Although yeasts and moulds can survive in acidic $\mathrm{pH}$, their numbers were decimated considerably with the addition of $500 \mu \mathrm{g} / \mathrm{ml}$ of chitosan to the growth medium. Saccharomyces cerevisiae for example at $\mathrm{pH} 3.0$ was reduced to $1.35-1.59 \log _{10} \mathrm{CFU} / \mathrm{ml}$ compared to $4.25-4.46 \log _{10} \mathrm{CFU} / \mathrm{ml}$ at $\mathrm{pH} 5.8$. Saccharomyces cerevisiae at $\mathrm{pH} 3.5$ was reduced by 3log cycles by DCMPA (Mw, 558 KDA) compared to less than $1 \log$ cycle reduction at $\mathrm{pH}$ 5.8. 
Table 1: Effect of $\mathrm{pH}$ on the Antibacterial Activity of Chitosans based on Colony Counts ( $\left.\mathrm{LOG}_{10} \mathrm{CFU} / \mathrm{ml}\right)^{1}$ on Gram-positive Bacteria

\begin{tabular}{|c|c|c|c|c|c|}
\hline \multirow[t]{2}{*}{ Microorganisms(Bacteria) } & \multicolumn{5}{|c|}{$\mathbf{p H}$} \\
\hline & 3.0 & 3.5 & 4.0 & 5.0 & 5.8 \\
\hline \multicolumn{6}{|l|}{ Staphylococcus aureus } \\
\hline Initial & 7.06 & 7.06 & 7.06 & 7.06 & 7.06 \\
\hline Control & 9.21 & 9.24 & 9.26 & 9.28 & 9.31 \\
\hline DMPAC (152) & $1.23 \mathrm{e}$ & $1.56 \mathrm{~d}$ & $2.08 \mathrm{c}$ & $2.25 b$ & $3.02 \mathrm{a}$ \\
\hline DMPCA(338) & $1.58 \mathrm{~d}$ & $1.76 \mathrm{c}$ & $2.30 \mathrm{~b}$ & $3.36 \mathrm{a}$ & $2.38 \mathrm{~b}$ \\
\hline $\operatorname{DPMCA}_{(2)}(418)$ & $1.49 \mathrm{~d}$ & $1.65 \mathrm{c}$ & $2.47 \mathrm{~b}$ & $3.31 b$ & $4.23 \mathrm{a}$ \\
\hline $\operatorname{DMCPA}(550)$ & $1.54 \mathrm{~d}$ & $1.72 \mathrm{c}$ & $2.65 b$ & $2.68 b$ & $5.04 \mathrm{a}$ \\
\hline DCMPA(558) & $1.76 \mathrm{c}$ & $1.81 \mathrm{c}$ & $2.82 \mathrm{~b}$ & $2.94 \mathrm{~b}$ & $5.26 \mathrm{a}$ \\
\hline \multicolumn{6}{|l|}{ Listeria monocytogenes } \\
\hline Initial & 6.45 & 6.45 & 6.45 & 6.45 & 6.45 \\
\hline Control & 8.60 & 8.76 & 8.81 & 8.85 & 8.89 \\
\hline DMPAC (152) & $\mathrm{ND}^{2 \mathrm{~b}}$ & $\mathrm{NDb}$ & $\mathrm{NDb}$ & $\mathrm{NDb}$ & $2.26 b$ \\
\hline DMPCA (338) & $\mathrm{NDb}$ & $\mathrm{NDb}$ & $\mathrm{NDb}$ & $\mathrm{NDb}$ & $2.48 \mathrm{a}$ \\
\hline $\operatorname{DPMCA}_{(2)}(418)$ & $\mathrm{NDb}$ & $\mathrm{NDb}$ & $\mathrm{NDb}$ & $\mathrm{NDb}$ & $2.53 \mathrm{a}$ \\
\hline DMCPA (550) & $\mathrm{NDb}$ & $\mathrm{NDb}$ & $\mathrm{NDb}$ & $\mathrm{NDb}$ & $2.60 \mathrm{a}$ \\
\hline DCMPA(558) & $\mathrm{NDb}$ & $\mathrm{NDb}$ & $\mathrm{NDb}$ & $\mathrm{NDb}$ & $2.73 \mathrm{a}$ \\
\hline \multicolumn{6}{|l|}{ Bacillus subtilis } \\
\hline Initial & 6.30 & 6.30 & 6.30 & 6.30 & 6.30 \\
\hline Control & 7.85 & 7.90 & 9.94 & 8.01 & 8.12 \\
\hline DMPAC (152) & NDc & NDc & NDc & $1.13 b$ & $2.15 \mathrm{a}$ \\
\hline DMPCA (338) & NDc & NDc & NDc & $1.24 \mathrm{~b}$ & $2.20 \mathrm{a}$ \\
\hline $\operatorname{DPMCA}_{(2)}(418)$ & $\mathrm{NDb}$ & $\mathrm{NDb}$ & $\mathrm{NDb}$ & $\mathrm{NDb}$ & $324 a$ \\
\hline DMCPA (550) & $\mathrm{NDb}$ & $\mathrm{NDb}$ & $\mathrm{NDb}$ & $\mathrm{NDb}$ & $3.58 \mathrm{a}$ \\
\hline DCMPA (558) & $1.18 \mathrm{c}$ & $1.21 \mathrm{c}$ & NDd & $2.05 \mathrm{~b}$ & $4.06 \mathrm{a}$ \\
\hline \multicolumn{6}{|l|}{ Lactobacillus casei } \\
\hline Initial & 6.75 & 6.75 & 6.75 & 6.75 & 6.75 \\
\hline Control & 8.23 & 8.67 & 8.89 & 8.99 & 9.24 \\
\hline DMPAC (152) & $1.08 \mathrm{e}$ & $1.15 \mathrm{~d}$ & $1.21 \mathrm{c}$ & $1.56 \mathrm{~b}$ & $2.60 \mathrm{a}$ \\
\hline DMPCA (338) & $1.43 \mathrm{~d}$ & $1.49 \mathrm{c}$ & $1.48 \mathrm{c}$ & $1.78 b$ & $2.87 \mathrm{a}$ \\
\hline $\operatorname{DPMCA}_{(2)}(418)$ & $1.62 \mathrm{~d}$ & $1.73 \mathrm{c}$ & $1.85 \mathrm{~b}$ & $1.91 \mathrm{~b}$ & $3.52 \mathrm{a}$ \\
\hline DMCPA (550) & $1.65 \mathrm{~d}$ & $1.72 \mathrm{c}$ & $1.96 \mathrm{~b}$ & $1.98 \mathrm{~b}$ & $3.49 \mathrm{a}$ \\
\hline DCMPA (558) & $2.03 \mathrm{~d}$ & $2.10 \mathrm{c}$ & $2.16 \mathrm{~b}$ & $2.20 \mathrm{~b}$ & $4.03 \mathrm{a}$ \\
\hline \multicolumn{6}{|l|}{ Lactobacillus plantarum } \\
\hline Initial & 6.71 & 6.71 & 6.71 & 6.71 & 6.71 \\
\hline Control & 8.76 & 8.85 & 8.91 & 8.96 & 9.09 \\
\hline DMPAC (152) & NDd & NDd & $2.05 \mathrm{c}$ & $2.17 b$ & $2.52 \mathrm{a}$ \\
\hline DMPCA (338) & NDd & $1.24 \mathrm{~d}$ & $1.56 \mathrm{c}$ & $2.89 b$ & $3.17 \mathrm{a}$ \\
\hline $\operatorname{DPMCA}_{(2)}(418)$ & $1.58 \mathrm{~d}$ & $1.72 \mathrm{c}$ & $1.84 \mathrm{~b}$ & $2.92 b$ & $3.86 \mathrm{a}$ \\
\hline $\operatorname{DMCPA}(550)$ & $2.57 \mathrm{e}$ & $2.68 \mathrm{~d}$ & $3.04 \mathrm{c}$ & $3.15 b$ & $4.10 \mathrm{a}$ \\
\hline DCMPA (588) & $3.06 \mathrm{~cd}$ & $3.13 \mathrm{c}$ & $3.19 \mathrm{c}$ & $3.40 \mathrm{~b}$ & $4.33 \mathrm{a}$ \\
\hline
\end{tabular}

NOTE: $a-e$ Means with different letters within a row indicate significant difference $(p<0.05) ; 1$. Viable cells after incubation without (control) and with $0.05 \%$ chitosan for 24 h at $37^{\circ} \mathrm{C} ; 2 . \mathrm{ND}=$ Not detected. Figures in parathenses are Molecular weight

The yeast Pichia fermentans was similarly affected by $\mathrm{pH}$. At $\mathrm{pH} 3.0$ the log reduction was in the range $1.48-2.20 \log _{10} \mathrm{CFU} / \mathrm{ml}, \quad 2.31-2.50 \log _{10} \mathrm{CFU} / \mathrm{ml}$ at $\mathrm{pH} 3.5,2.62-2.72 \log _{10} \mathrm{CFU} / \mathrm{ml}$ at $\mathrm{pH} 4.0,2.70-$ $2.85 \log _{10} \mathrm{CFU} / \mathrm{ml}$ at $\mathrm{pH} \quad 5.0$ and 3.17$3.41 \log _{10} \mathrm{CFU} / \mathrm{ml}$, at $\mathrm{pH} 5.8$. The $\log$ reduction in counts of this organism decreased with increasing $\mathrm{pH}$ (Table 3).

The growth of the moulds Penicillium expansum, Aspergillus niger and Rhizopus stolonifer were affected by $\mathrm{pH}$ on the addition of $500 \mu \mathrm{g} / \mathrm{ml}$ of chitosans to the medium. In the control experiment with no chitosan, Penicillium expansum grew from 5.20-8.56Log10CFU/ml at $\mathrm{pH} 3.0$ and 5.20-9.02 $\mathrm{log}$ $\mathrm{cfu} / \mathrm{ml}$ at $\mathrm{pH}$ 5.8. With chitosan addition Penicillium expansum showed 1.11-1.87 viable cell $\log _{10}$ number at $\mathrm{pH} 3.0,1.51-2.17(\mathrm{pH} 3.5), 3.02-3.31(\mathrm{pH} 4.0)$, 3.35-3.63 (pH 5.0) and 4.02-4.17 $(\mathrm{pH} \mathrm{5.8)} \mathrm{in} \mathrm{that}$ order. The log reduction of Aspergillus niger was greater at low $\mathrm{pH}$ values. With DMPAC chitosan, a $3.23 \log _{10}$ reduction occurred at $\mathrm{pH} 3.0$ compared to $1.30 \log _{10}$ reduction at $\mathrm{pH}$ 5.8. Rhizopus Stolonifer was reduced to non-detectable levels by DMPAC chitosan at $\mathrm{pH}$ 3-3.5.This mould was more sensitive to chitosan $(500 \mu \mathrm{g} / \mathrm{ml})$ at all $\mathrm{pH}$ compared to Penicillium expansum and Aspergillus niger. At $\mathrm{pH}$ 5.8 the range of $\log$ reduction was 4.02-4.17, 4.0-4.50 and 2.30-2.86 for Penicillium expansum, Aspergillus niger and Rhizopus stolonifer respectively (Table 3 ).

The antimicrobial activity of chitosan was found to increase with decreasing $\mathrm{pH}$. Decrease in $\mathrm{pH}$ increased solubility by forming polycationic polymer. This is due to the fact that amino groups of chitosan become ionized at $\mathrm{pH}$ below 6 and carry a positive charge. (Muzzarelli, 1973).

The greater the positive charge the more active this polymer becomes. It is worthwhile to note that growth of Listeria monocytogenes and Bacillus subtilis were completely deactivated or suppressed by $500 \mu \mathrm{g} / \mathrm{ml}$ chitosan at or below $\mathrm{pH} 5.5$. The presence of Listeria monocytogenes in foods has become a concern in recent years. 
Table 2: Effect of $\mathrm{pH}$ on Antibacterial Activity of Chitosans based on Colony Counts $\left(\mathrm{LOG}_{10} \mathrm{CFU} / \mathrm{ml}\right)^{1}$ on Gram-negative Bacteria

\begin{tabular}{|c|c|c|c|c|c|}
\hline \multirow[t]{2}{*}{ MGicroorganims (Bacteria) } & \multicolumn{5}{|c|}{$\mathrm{pH}$} \\
\hline & 30 & 3.5 & 40 & 50 & 58 \\
\hline \multicolumn{6}{|l|}{ Eatheriolis coti $0157 \mathrm{HT}$} \\
\hline Initival & 7.67 & 7.67 & 7.67 & 7.67 & 7.67 \\
\hline Consol & 3.88 & 8.94 & 9.10 & 9.45 & 9.62 \\
\hline DMPAC (152) & 2050 & 3.300 & 4.650 & $5.32 \mathrm{~b}$ & 5.682 \\
\hline DMPCA (335) & 2.130 & 3.430 & $4.78 \sigma$ & $5.65 b$ & $5.35 \mathrm{z}$ \\
\hline$D_{P M C A} A_{(2)}(415)$ & 2280 & $3.51 d$ & $4.81 \mathrm{e}$ & $5.76 \mathrm{~b}$ & 5.912 \\
\hline DMCPA $(550)$ & 2360 & 3.590 & $4.86 \mathrm{e}$ & $5.79 \mathrm{ab}$ & 5952 \\
\hline DCMPA (558) & $242 d$ & $3.62 e$ & $4.92 \mathrm{~b}$ & $5.83 \mathrm{a}$ & $5.90 \mathrm{z}$ \\
\hline \multicolumn{6}{|l|}{ Solnonelle typkinterient } \\
\hline Initial & 7.57 & 7.57 & 7.57 & 7.57 & 7.57 \\
\hline Consol & 9.23 & 9.35 & 9.48 & 9.49 & 9.54 \\
\hline DMPAC (152) & 2050 & $3.41 d$ & $4.73 c$ & $5.44 b$ & $3.74 \mathrm{z}$ \\
\hline DMPCA (338) & 2220 & 3.460 & 4.350 & $5.57 b$ & $5.78 \mathrm{z}$ \\
\hline $\operatorname{DPMCA}_{[23}(418)$ & 2310 & $3.62 e$ & $4.90 b$ & $5.69 \%$ & $6.01 \mathrm{z}$ \\
\hline DMCPA $(590)$ & 2560 & 3.790 & 4960 & $5.75 b$ & $6.21 \mathrm{z}$ \\
\hline $\mathrm{DCMPA}(528)$ & 2.610 & $4.52 e$ & 4980 & 5.926 & $632 \mathrm{z}$ \\
\hline \multicolumn{6}{|l|}{ 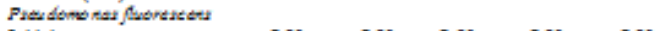 } \\
\hline Initial & 7.82 & 7.32 & 7.82 & 7.82 & 7.82 \\
\hline Consol & 9.25 & 9.33 & 9.38 & 9.54 & 9.56 \\
\hline DMPAC (152) & $\mathrm{NDe}$ & Ne & $1.15 \mathrm{~b}$ & $1.15 \mathrm{~b}$ & $2.18 \mathrm{a}$ \\
\hline DMPCA (335) & NDd & 1.140 & NDed & 1.832 & 1942 \\
\hline $\mathrm{DPMCA}_{(23)}(418)$ & 1.340 & 1.450 & $1.52 e$ & $1.95 \mathrm{~b}$ & $3.10 \mathrm{z}$ \\
\hline DMCPA $(550)$ & $1.420 d$ & 1.510 & NDo & $2.19 \mathrm{~b}$ & 3.422 \\
\hline DCMPA (558) & 1.48 & 1.58 & 1.64 & 2.33 & 3.58 \\
\hline \multicolumn{6}{|l|}{ 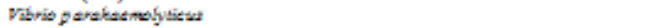 } \\
\hline Initivel & 6.70 & 6.70 & 6.70 & 6.70 & 6.70 \\
\hline Consol & 395 & 899 & 9.21 & 9.36 & 9.48 \\
\hline DMPAC (152) & $\mathrm{NDb}$ & $\mathrm{NDb}$ & $\mathrm{NDb}$ & $\mathrm{NDb}$ & $3.06 \mathrm{a}$ \\
\hline DMPCA (338) & NDo & 1.340 & $1.44 \phi$ & $1.55 \mathrm{~b}$ & $3.22 \mathrm{z}$ \\
\hline DPMCA $A_{[32}(418)$ & NDo & 1.428 & $1.62 \mathrm{~b}$ & $1.74 b$ & 3.412 \\
\hline DMCPA $(590)$ & NDo & $1.53 d$ & 1.650 & $1.75 \mathrm{~b}$ & $3.57 \mathrm{a}$ \\
\hline DCMPA (558) & NDo & $1.61 d$ & $1.71 \mathrm{e}$ & $1.83 \mathrm{~b}$ & $4.14 \mathrm{z}$ \\
\hline
\end{tabular}

NOTE: $a-e$ Means with different letters within a row indicate significant difference $(p<0.05)$. 1. Viable cells after incubation without (control) and with $0.05 \%$ chitosan for 24 h at $37^{\circ} \mathrm{C} 2 . \mathrm{ND}=$

Not detected, Figures in parathenses are Molecular weight

Table 3: Effect of $\mathrm{pH}$ on Antifungal Activity of Chitosans Based on Colony Counts $\left(\log _{10} \mathrm{CFU} / \mathrm{ml}\right)^{1}$ on Yeast and Moulds

\begin{tabular}{|c|c|c|c|c|c|}
\hline \multirow{2}{*}{ Microorganisms (Fugi) } & \multicolumn{5}{|c|}{$\mathrm{pH}$} \\
\hline & 3.0 & 3.5 & 4.0 & 5.0 & 5.8 \\
\hline \multicolumn{6}{|l|}{ 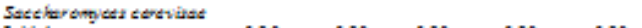 } \\
\hline Initial & 530 & 5.30 & 5.30 & 5.30 & 5.30 \\
\hline Consol & 7.87 & 7.89 & 8.03 & 7.96 & 3.09 \\
\hline DMPAC (152) & 1.350 & $209 d$ & 3.160 & $3.41 \mathrm{~b}$ & $4.25 \mathrm{z}$ \\
\hline DMPCA (335) & 1.4660 & 2150 & $3.20 c$ & $3.45 \mathrm{~b}$ & $430 \mathrm{z}$ \\
\hline$D_{P M C A} A_{129}(418)$ & 1.510 & 2.190 & $3.26 c$ & $3.45 \mathrm{~b}$ & $4.37 \mathrm{z}$ \\
\hline DMCPA $(950)$ & 1.560 & 2250 & $3.30 c$ & 3.536 & 4.402 \\
\hline DCMPA (598) & 1.590 & $230 d$ & $3.39 c$ & $3.5 \% \mathrm{~b}$ & $4.46 \mathrm{a}$ \\
\hline \multicolumn{6}{|l|}{ Pitkightemettan } \\
\hline Initival & 5.54 & 5.54 & 5.54 & 5.54 & 5.54 \\
\hline Control & 8.66 & 8.71 & 8.75 & 3.90 & 3.59 \\
\hline DMPAC (152) & 1.486 & $231 \mathrm{e}$ & $2.62 \mathrm{~b}$ & $2706 \mathrm{~b}$ & $3.17 \mathrm{z}$ \\
\hline DMPCA (338) & 1.520 & 2350 & $2.64 c$ & $274 \mathrm{~b}$ & $3.20 \mathrm{z}$ \\
\hline $\operatorname{DPMCA}_{[20}(418)$ & 2050 & $238 c$ & $264 b$ & $275 b$ & $3.25 \mathrm{z}$ \\
\hline DMCPA $(550)$ & 2110 & $242 d$ & $2.67 \mathrm{bc}$ & $281 \mathrm{~b}$ & $3.30 \mathrm{z}$ \\
\hline DCMPA (5S8) & 2200 & 2500 & $272 e$ & 2.556 & $3.41 \mathrm{z}$ \\
\hline \multicolumn{6}{|l|}{ 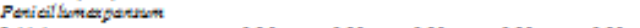 } \\
\hline Initiva! & 5.20 & 5.20 & 5.20 & 5.20 & 5.20 \\
\hline Consol & 8.56 & 3.68 & 8.78 & 8.96 & 9.02 \\
\hline DMPAC (152) & 1.110 & i.sid & $3,02 e$ & $3.35 \mathrm{~b}$ & $4.02 \mathrm{z}$ \\
\hline DMPCA (338) & 1.250 & 1.590 & $3.14=$ & $3.42 \mathrm{~b}$ & $405 \mathrm{a}$ \\
\hline $\mathrm{DPMCA}_{(20}(418)$ & $1.78 d 0$ & 1.850 & $3.18 c$ & 3.506 & $4.10 \mathrm{z}$ \\
\hline DMCPA $(590)$ & 1.830 & $203 d$ & 3.250 & $3.56 \mathrm{~b}$ & 4.112 \\
\hline DCMPA (518) & 1.870 & $2.17 d$ & $3.31 \mathrm{e}$ & $3.63 \mathrm{ab}$ & $4.17 \mathrm{z}$ \\
\hline \multicolumn{6}{|l|}{ 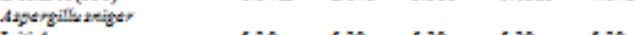 } \\
\hline Initiva! & 530 & 5.30 & 5.30 & 5.30 & 5.30 \\
\hline Consol & 8.75 & 8.39 & 9.26 & 9.31 & 9.45 \\
\hline DMPAC (152) & 2070 & 2630 & $3.20 c$ & $3.61 \mathrm{ab}$ & $4.00 \mathrm{z}$ \\
\hline DMPCA (333) & 2180 & 2.660 & $3.26 \mathrm{c}$ & $3.65 \mathrm{~b}$ & $4.19 \mathrm{z}$ \\
\hline DPMCA A $(418)$ & 2250 & 2798 & $3.31 \mathrm{e}$ & $3.69 \%$ & $4.26 \mathrm{z}$ \\
\hline DMCPA $(550)$ & 2370 & $287 d$ & $3.45 \mathrm{e}$ & $3.74 \mathrm{~b}$ & $4.34 \mathrm{z}$ \\
\hline DCA.PA(525) & 2480 & 2940 & $3.58 c$ & $3.5 .6 \mathrm{~b}$ & $4.50 \mathrm{z}$ \\
\hline \multicolumn{6}{|l|}{ 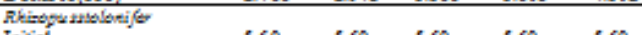 } \\
\hline Initiví & 5.60 & 5.60 & 5.60 & 5.60 & 5.60 \\
\hline Consol & 9.34 & 9.39 & 9.46 & 9.35 & 9.47 \\
\hline DMPAC (152) & NDd & NDd & $1.16 \mathrm{bc}$ & $1.41 \mathrm{~b}$ & $275 \mathrm{z}$ \\
\hline DMPCA (335) & NDde & 1.120 & $1.18 \mathrm{e}$ & 1.536 & $278 \mathrm{z}$ \\
\hline$D^{D P M C A} A_{22}(418)$ & 1.230 & $1.36 c$ & $1.44 z$ & 1.570 & 2822 \\
\hline DMCPA $(\$ S 0)$ & $1.29 \mathrm{~d}$ & $1.42 e$ & $1.30 d$ & 1.636 & $286 \mathrm{a}$ \\
\hline DCMPA $(598$ & 1350 & 1.158 & 1350 & $1.71 \mathrm{~b}$ & $230 \mathrm{z}$ \\
\hline
\end{tabular}

NOTE: $a$-e Means with different letters within a row indicate significant difference $(p<0.05)$. 1. Viable cells after incubation without (control) and with $0.05 \%$ chitosan for 24 h at $37^{\circ} \mathrm{C}$. 2. ND

= Not detected, Figures in parathenses are Molecular weight .

Confirmed outbreaks of human Listeriosis have been associated with consumption of contaminated foods from plant and animal sources. The ability of Listeria monocytogenes to proliferate at refrigeration temperatures and cause serious illness have been reported (Ahamad and Marth, 1989), Thus, a significant health hazard could result by consumption of foods contaminated with this organism but possibly could be reduced or prevented by proper chitosan treatment. The $\mathrm{pH}$ values of tropical fruit juices are usually acidic and range between 3.54 and 5.56. At low pH, both Escherichia coli 0157: H7 and Salmonella typhimurium survive for several days, especially when stored at refrigeration temperatures as reported by McClure and Hall (2000); Youssuf and Munir (2007). Thus the acidic nature of unpasteurized fruit juices does not ensure its safety as some pathogens may survive for extended periods of time and cause disease (CDC, 1997). While some pathogens gradually died off with chitosan addition at low $\mathrm{pH}$, others were killed rapidly showing the potential efficacy for use of chitosan for fruit juice preservation.

Conclusion: The bioactivity of chitosan was affected by acidic $\mathrm{pH}$ showing the inhibition and total killing of food-borne bacteria and fungi which are either pathogenic or spoilage organisms. Thus the results of the present study clearly show that application of chitosan to acidic foods such as fruit juices will enhance its effectiveness as a natural preservative.

\section{REFERENCES}

Ahamad, N; Marth, EH (1989). Behaviour of Listeria monocytogenes at 7,13,21 and $35^{\circ} \mathrm{C}$ in tryptose broth acidified with acetic, citric, or lactic acid. J. Food Protect. 52: 688 - 695.

CDC (1997). Outbreak of Escherichia coli 0157:H7 infection and criptosporidiosis associated with drinking unpasteurized apple cider-Connecticut and New York. J. American Med. Assoc.277: 781-787.

Hsu, SC; Don, TM; Chiu, WY (2002). Free radical degradation of chitosan with potassium persulfate. Polymer Degrad. Stab.755: 73 - 83 .

Kurita, K (1998). Chemistry and application of chitin and chitosan. Polymer Degrad. Stab.59: 117 120.

Li, Q; Dunn, EJ; Grandmaison, EW; Goosen, MF (1992). Applications and properties of chitosan. J. Bioactive Comp. Polymers. 7: 370 - 397.

Mcclure, PJ; Hall, S (2000). Survival of Escherichia coli in foods. J. Appl. Microbiol.supplement .88: $61 \mathrm{~s}-70 \mathrm{~s}$

Muzzarelli, RAA (1973). Natural Chelating Polymers Pergamon Press, Oxford, U.K. 
No, HK; Meyers, SP (1989). Crawfish chitosan as a coagulant in recovery of organic compounds from seafood processing streams. J. Agric. Food Chem.37: $580-583$.

Omogbai, BA; Ikenebomeh, MJ (2013). Antimicrobial and toxicological evaluation of food grade chitosan from crab (Callinectes sapidus). NISEB J. 13 (1\&2): 23-28.

Omogbai, BA; Ikenebomeh, MJ (2016).Sub-chronic toxicity study of a characterized food grade chitosan from crab (Callinectes sapidus).NISEB J. 16 (1): 34-44.
Sakthivel, D; Vijayakumar, N; Anandan, V (2015). Extraction of chitin and chitosan from Mangrove crab (Sesarmaplicatum) from Thengaithittu estuary Pondicherry Southeast coast of India. Intl. J. Pharm. Pharmceu. Res. 4(1); 12-24.

Yossuf, AA; Munir, MA (2007). Experimental studies on the potential for acid tolerance, growth and survival of Salmonella enteric Serovar Typhimurium and Escherichia coli 0157: $\mathrm{H} 7$ in orange juices. Adv. Biol. Res.1 (3-4): 99107. 